\title{
Artificial Immunity-Based Discovery for Popular Information in WEB Pages
}

\author{
Caiming Liu, Xiaojie Liu, Tao Li, Lingxi Peng, Jinquan Zeng, and Hui Zhao
}

\author{
School of Computer Science, \\ Sichuan University \\ 610065 Chengdu, China \\ liucaiming@gmail.com
}

\begin{abstract}
An artificial immunity-based discovery method for popular information is proposed. Principles of evolution and concentration of antibodies in artificial immune system are simulated. Key words in web pages are extracted and simulated as antibody and antigen. Antibodies are evolved and excreted dynamically. Concentration of antibodies is computed to attain accurately the degree of popular measurement in quantity. The proposed method improves the intelligent degree of information discovery and provides a new way to discover WEB information.
\end{abstract}

Keywords: artificial immune, popular information, information discovery, antibody, concentration.

\section{Introduction}

WEB pages comprise a great deal of information. How to extract useful knowledge from WEB pages is focused by researchers. Intelligent methods are needed to compute degree of popular measurement in quantity.

Artificial immune system (AIS) which simulates the characters of learning and self-adaptation [1,2] of biological immune system uses the way of clone selection and mutation principles to recognize foreign harmful antigens [3, 4]. Furthermore, if an antigen damages body greatly, the antibody which recognizes the antigen excretes many similar antibodies [5, 6]. Concentration of this kind of antibody will increase quickly. The antibody concentration can be used to judge the harmful measurement of relative antigen [7].

In this paper, the former principles is used for reference. Key words in the WEB pages are extracted to be simulated as antibodies which will be evolved in our model automatically. Their concentration is computed to judge the degree of popular measurement of WEB information.

The rest of the paper is organized as follows. In Section 2, the proposed principle is introduced. In Section 3, simulations and experimental results are showed. Finally, Sections 4 contains our conclusions. 


\section{Proposed Principle of Information Discovery}

\subsection{Information Simulation}

Key words are extracted to find popular information hid in WEB pages. The key words extracted from WEB pages are simulated as antigens. Let $U=\bigcup_{i=1}^{+\infty} G^{i}$ be the state space of antigen, where $G$ is Chinese or English word library.

Let antibody represent information of key words. Antibody is defined as $D=\langle$ Keyword, age, count,type $\rangle . D$ is a four-tube group. It is consisted of key word information (keyword), the live generations of antibody (age), the amount of antigens which antibody matches (count) and the classification of the information (type).

Antibody is consisted of mature antibody and memory antibody. Mature antibody needs to match enough number $(\delta)$ of antigens in its lifecycle $\lambda_{M}$ to evolve into memory antibody. Or else, it dies. The type of antibody which is extracted in the first time is the key word self. Mature antibody $M$ is described in equation (1).

$$
M=\left\{x \mid x \in D \wedge x \text {.age }<\lambda_{M} \wedge x \text {.count }<\delta\right\}
$$

Where, $\lambda_{M}$ is the lifecycle of antibody, $\delta$ is threshold of evolution.

Memory antibody $R$ is evolved by mature antibody. It denotes that information emerges frequently recently. Memory antibody can live in a long lifecycle $\lambda_{R}$ even it doesn't match any antigens in long period of time.

\subsection{Evolution of Antibody}

Mature antibody is extracted from WEB pages newly or generated by clone selection to recognize new information. If mature antibody can not match enough antigens in its lifecycle, it tends toward death. Once its matching count arrives at the threshold $\delta$ (i. e. count $\geq \delta$ ), it will be activated and evolved into memory antibody. During the period $\lambda_{M}$, some mature antibodies match few antigens. This kind of antibody will die soon. If a key word appears in a high frequency, matching count of its relative antibody will be accumulated to threshold $\delta$ sharply. Its relative antibody will be evolved into memory quickly. This represents the key word is popular.

Memory antibody denotes a key word is popular. It has long lifecycle $\lambda_{R}$. However, $\lambda_{R}$ is not infinite. The past popular information may not appear again or emerge in low frequency in future. When a memory antibody can not match any key words in the period $\lambda_{R}$, its affinity count decreases in a time interval $T_{\text {interval }}$ (count is decreased by 1$)$. When the affinity is equal to 0 , memory antibody is degenerated as mature antibody. If the memory antibody matches antigen again, its affinity increase ( count is added by 1). If it becomes popular again, its affinity ascends quickly. 


\subsection{Degree Computation of Popular Measurement}

To discover relative information, memory antibody excretes new mature antibody rapidly by the way of copy, mutation, and etc. By computing the concentration of antibody, the popular degree of relative information can be worked out. According to the type of antibody, its concentration is obtained. The concentration represents the popular degree. Equation (2) describes antibody concentration.

$$
h r\left(r_{i}\right)=c\left(\frac{2}{\left.1+e^{-\left(\sum r_{i} \text { type }+\sum m_{i}\right. \text { type }}\right)}-1\right)
$$

Where, $h r\left(r_{i}\right)$ denotes the popular degree of information similar to memory antibody $r_{i}$, the lager the $h r\left(r_{i}\right)$, the relative key word is more popular; $m_{i}$ is mature antibody and in the same kind of $r_{i} ; c$ is a constant number, $c>0$.

\section{Experimental Results}

In our experiments, two portal web sites and two BBSes are chosen to test the efficiency of proposed method. Choices of monitor spots are shown in table 1 . The four columns of table 1 represent the following parameters respectively: name of web site, lifecycle $\lambda_{M}$ (see section 2.1) of mature antibody, lifecycle of memory antibody $\lambda_{R}$ (see section 2.1) and degeneration time interval $T_{\text {int erval }}$ (see section 2.2) of memory antibody.

Table 1. Monitor spots and configuration of parameters

\begin{tabular}{cccc}
\hline Monitor spot & $\lambda_{M}$ & $\lambda_{R}$ & $T_{\text {int } \text { erval }}$ \\
\hline Web site 1 & One week & One day & Half day \\
\hline Web site 2 & One week & One day & Half day \\
\hline BBS 1 & Four days & Five hours & 1.5 hours \\
\hline BBS 2 & Four days & Two hours & 1.5 hours \\
\hline
\end{tabular}

A great lot of key words are extracted from pages of former web sites. These key words are simulated as antibodies. We monitor the four web sites from September to November in 2006. Through evolvement of antibody, concentration of antibody is worked out. According to the concentration, we obtain the popular degree of some information by equation (2), as is shown in figure 1. The larger popular degree is, the more popular the key word is. It is National Day of Chinese in October 1. So, people pay much attention to the key word "national day" whose concentration ascends sharply. 


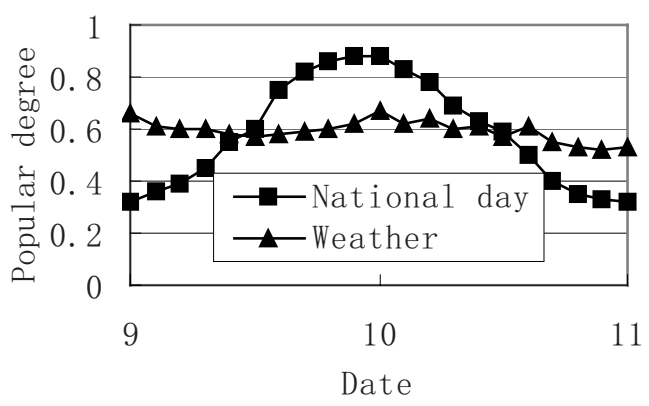

Fig. 1. The measurement of popular information

\section{Conclusion}

In this paper, key words in WEB pages are simulated as antibodies and antigens. Antibody evolution and clone selection principles in AIS are used to evolve antibodies dynamically. Degeneration of memory antibody is proposed to simulate popular information degradation. Concentration of antibody forms automatically. The concentration represents popular degree of information in quantity. The experimental results show that our method can get the popular degree of information efficiently.

Acknowledgments. This work was supported by the National Natural Science Foundation of China under Grant No.60373110, No.60573130 and No.60502011, the National Research Foundation for the Doctoral Program of Higher Education of China under Grant No.20030610003, the New Century Excellent Expert Program of Ministry of Education of China under Grant No. NCET-04-0870, and the Innovation Foundation of Sichuan University under Grant No.2004CF10.

\section{References}

1. Li, T.: Computer Immunology[M]. Publishing House of Electronics Industry, Beijing (2004)

2. Forrest, S., Hofmeyr, S. A., Somayaji, A.: Computer immunology[J]. Communications of the ACM, Vol. 40(10) (1997) 88-96

3. De Castro, L. N., Timmis, J. I.: Artificial Immune Systems: A Novel Computational Intelligence Approach[M]. London: Springer-Verlag, 2002

4. Hofmeyr, S. A., Forrest, S.: Architecture for an Artificial Immune System[J]. Evolutionary Computation, Vol. 8(4) (2000) 443-473

5. Hofmeyr, S. A., Forrest, S.: Immunity by design: an artificial immune system[C]. In: Genetic Evolutionary Computation Conf, San Francisco, CA, (1999) 1289-1296

6. Timmis, J., Neal, M., Hunt, J.: An artificial immune system for data analysis. Biosystems, Vol. 55(1/3) (2000) 143-150

7. Li, T.: An immunity based network security risk estimation. Science in China(Series E), Vol. 35(8) (2005) 798-816 